\title{
Nonrelativistic quantum dynamics on a cone with and without a constraining potential
}

C. Filgueiras, 1, a) E. O. Silva, 2, b) and F. M. Andrade, (c)

1) Departamento de Física, Universidade Federal de Campina Grande, Caixa Postal 10071, 58109-970 Campina Grande-PB, Brazil

${ }^{2)}$ Departamento de Física, Universidade Federal do Maranhão, Campus Universitário do Bacanga, 65085-580 São Luís-MA, Brazil

3) Departamento de Matemática e Estatística, Universidade Estadual de Ponta Grossa, 84030-900 Ponta Grossa-PR, Brazil

(Dated: 6 June 2018)

In this paper we investigate the bound state problem of nonrelativistic quantum particles on a conical surface. This kind of surface appears as a topological defect in ordinary semiconductors as well as in graphene sheets. Specifically, we compare and discuss the results stemming from two different approaches. In the first one, it is assumed that the charge carriers are bound to the surface by a constraining potential, while the second one is based on the Klein-Gordon type equation on surfaces, without the constraining potential. The main difference between both theories is the presence/absence of a potential which contains the mean curvature of a given surface. This fact changes the dependence of the bound states on the angular momentum $l$. Moreover, there are bound states that are absent in the Klein-Gordon theory, which instead appear in the Schrödinger one.

PACS numbers: 03.65.Ge, 03.65.Db, 98.80.Cq, 11.27.+d

\footnotetext{
a) Electronic mail: cleversonfilgueiras@yahoo.com.br

b)Electronic mail: edilbertoo@gmail.com

c) Electronic mail: fmandrade@uepg.br
} 


\section{INTRODUCTION}

Quantum mechanics in two dimensions is a subject of great interest and has received much

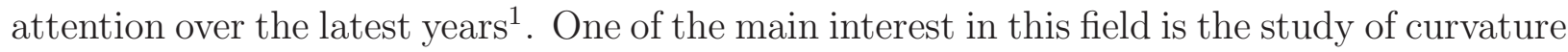
effects on a two dimensional electron gas (2DEG). Curvature effects on the conductance ${ }^{2}-\underline{\underline{5}}$ and curvature effects on the magnetization and persistent currents $\underline{\underline{6}}$ are some phenomena studied in 2DEG's. Usually, before considering any application, the bound state problems of non planar 2DEG's are investigated $\underline{\underline{7}} \underline{\underline{-9}}$.

In 1981, R.C.T da Costa published a paper deriving the Schrödinger equation of a free particle constrained to move on a curved surface 10 (the same problem in presence of external magnetic and electric fields was addressed in Ref. 11 by Ferrari and Cuoghi). He considered the constraint imposed by the action of an external potential, limiting the motion of noninteracting electrons to a thin interface with constant thickness $d$. This way, the normal modes are separated from the ones along the surface, in such way the Schrödinger equation for the normal modes(perpendicular to the interface) is

$$
-\frac{\hbar^{2}}{2 M} \frac{\partial^{2} \chi_{n}}{\partial q_{n}}+V\left(q_{n}\right) \chi_{n}=E_{n} \chi_{n}
$$

where $q_{n}$ is the normal coordinate, and $V\left(q_{n}\right)$ is the potential that confines the particle to the thin interface. The longitudinal modes are obtained from the Schrödinger equation for a spinless particle written in the coordinates of a curved surface, that is

$$
\frac{1}{2 M}\left[-\frac{\hbar^{2}}{\sqrt{g}} \partial_{\mu}\left(\sqrt{g} g^{\mu \nu} \partial_{\nu}\right)\right] \Psi+V_{\mathrm{daCosta}} \Psi=E \Psi,
$$

where $g^{\mu \nu}$ is the contravariant component of the metric tensor of the manifold, $g=\operatorname{det} g_{\mu \nu}$, and $V_{\mathrm{daCosta}}$ is a scalar geometric potential given by

$$
V_{\mathrm{daCosta}}=-\frac{\hbar^{2}}{2 M}\left(\mathrm{M}^{2}-\mathrm{K}\right)
$$

In this expression, $\mathrm{M}=\left(\kappa_{1}+\kappa_{2}\right) / 2$ is the mean curvature, and $\mathrm{K}=\kappa_{1} \kappa_{2}$ is the Gaussian curvature of the surface, while $\kappa_{1}$ and $\kappa_{2}$ are the principal curvatures of the surface. The da Costa's theory is applied to a surface making $d \rightarrow 0$. In this theory, possible applications to a bilayer graphene were investigated in Refs. $\stackrel{12}{-14}$. Curvature-induced $p-n$ junctions in bent sheets were predicted in Ref. $\frac{12}{}$, while an analog quantum Hall effect due to the geometry of a helicoidal ribbon was reported in Ref. 13 . 
In Ref $\underline{15}$, the authors pointed out that the intrinsic second order Dirac theory on a surface in ordinary three-dimensional space exhibits a new scalar geometric potential. It is induced by the interaction between the intrinsic spin and the surface geometry. This term is absent in the Schrödinger theory based on the dimensional reduction framework of da Costa's approach. The main difference is the absence of confinement, expressed by Eq. (11). In $(2+1)$-dimensions, the squared Dirac equation is given by

$$
\left(-D_{t}^{2}+D_{\|}^{2}+\frac{1}{8} \mathrm{R}_{A B C D} \gamma^{A} \gamma^{B} \gamma^{C} \gamma^{D}-M^{2}\right) \Psi=0
$$

where $\Psi$ is the Dirac spinor field, $D_{\|}^{2}$ is the tangential surface component of the kinetic operator, the matrices $\gamma$ obey local Clifford algebra, and $\mathrm{R}_{A B C D}$ are the components of the Riemann curvature. The low energy limit of the massive Dirac theory, neglecting all the spin-connection terms, is given by the following Klein-Gordon (K-G) type equation

$$
\left(-\partial_{t}^{2}+\nabla_{\|}^{2}-\frac{1}{4} \mathrm{R}-M^{2}\right) \psi=0 .
$$

with $\psi$ being assumed as a definite spin-state, and $\nabla_{\|}$being the usual covariant derivative acting on a scalar function. We assume energy eigenstates and denote the total energy of the particle by $E$. Considering the first order in an $1 / M$-expansion and reinstating $\hbar$, the equation above will take the following form,

$$
-\frac{\hbar^{2}}{2 M} \nabla_{\|}^{2} \psi+\frac{\hbar^{2}}{4 M} \mathrm{R} \psi=E_{c} \psi .
$$

which is the Schöredinger equation corresponding to the K-G type equation above. In this equation, $E_{c}$ is the classical energy measure and $\mathrm{R}$ is the Ricci curvature scalar in the static surface. The spectrum of Eq. (4) and (6) are related by the relation

$$
E_{c}=E-M
$$

In two dimensions, $\mathrm{R}=2 \mathrm{~K}$, with $\mathrm{K}$ being the Gaussian curvature of a surface. Then, we see that the Schrödinger equation corresponding to the K-G equation shows the effective potential,

$$
V_{\text {geo }}=\frac{\hbar^{2}}{2 M} \mathrm{~K}
$$

for fermions in two dimensions. As pointed out in reference $\frac{15}{}, V_{\mathrm{daCosta}}$ and $V_{\text {geo }}$ have profound differences. While $V_{\text {daCosta }}$ is always attractive, while $V_{\text {geo }}$ can be either attractive or repulsive. Notice that the difference between both geometric potentials is that in $V_{\mathrm{daC} \text { osta }}$ 
there is a contribution from the mean curvature $\mathrm{M}$ while no such contribution is present in $V_{\text {geo }}$. In differential geometry, $\mathrm{K}$ is an intrinsic property, since it can be written solely in terms of the metric of a surface. The mean curvature $M$ depends on how a surface is immersed in $3 D$ and it is an extrinsic property. Since in the da Costa's approach one has confinement, it would be expected the geometrical potential showing a piece containing M.

In this work, we are interested in studying the bound state problem of nonrelativistic quantum particles constrained to move on a conical surface. This problem was studied before in reference $\frac{16}{}$. Here, we are going to investigate the same problem but now considering the Schöredinger equation which comes from the first order $1 / M$-expansion of the K-G equation. We are interested in comparing compare the bound states for a particle on a cone in both theories. We choose this particular geometry since it is associated to topological defects in ordinary semiconductors $\underline{17}-\underline{19}$ as well as in graphene sheets (multilayers) ${ }^{20}$. We will see that, by considering $V_{\text {geo }}$ instead of $V_{\text {daCosta }}$, the dependence of the bound states on the angular momentum $l$ is altered.

\section{THE CONICAL SURFACE}

Using polar coordinates $\rho$ and $\theta$, the following line element

$$
d s^{2}=d \rho^{2}+\alpha^{2} \rho^{2} d \theta^{2},
$$

describes a conical surface for $\rho \geq 0$ and $0 \leq \theta<2 \pi$, describes a conical surface. For $0<\alpha<1$ (deficit angle), the metric (9) describes an actual cone. Figure 1 shows the making of a cone from a planar sheet where an angular section was removed with posterior identification of the edges. If $\gamma$ is the angle that defines the removed section then the remaining surface corresponds to an angular sector of $2 \pi \alpha=2 \pi-\gamma$. By identification of the length of the circle without the sector, $2 \pi \alpha \rho$, with the length of the complete circle it turns out to be on the cone, $2 \pi \rho \sin \beta$, we get the relation

$$
\alpha=\sin \beta,
$$

where $2 \beta$ is the opening angle of the cone (see Fig. 1. The closer $\alpha$ gets to 1 (or, equivalently, $2 \beta$ to $\pi$ ) the flatter is the cone. For $\alpha=1$ the cone turns into a plane. If $\alpha>1$ (proficit angle), relation (9) still holds and the conical surface corresponds to the insertion of a sector (i.e. $2 \beta>\pi$ ). We call the resulting surface an anti-cone. 


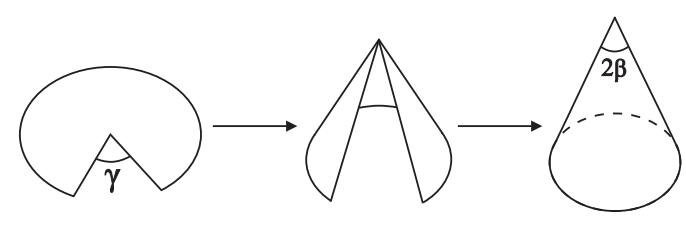

FIG. 1. Conical surface of angular deficit $\gamma$.

For a cone $e^{21}$,

$$
\mathrm{K}=\left(\frac{1-\alpha}{\alpha}\right) \frac{\delta(\rho)}{\rho}
$$

and

$$
\mathrm{M}=\frac{\sqrt{1-\alpha^{2}}}{2 \alpha \rho} .
$$

Notice that the $\delta$-function singularity in the Gaussian curvature corresponds cone tip. In the Schrödinger theory, a particle whose motion is confined to the conical surface is subjected to a resultant potential, given by

$$
V_{\mathrm{daCosta}}=-\frac{\hbar^{2}}{2 M}\left[\left(\frac{1-\alpha^{2}}{4 \alpha^{2} \rho^{2}}\right)-\left(\frac{1-\alpha}{\alpha}\right) \frac{\delta(\rho)}{\rho}\right] .
$$

There is also a contribution from a inverse squared distance interaction. The Schrödinger equation for the particle, in this case, is

$$
-\frac{\hbar^{2}}{2 M}\left[\frac{1}{\rho} \frac{\partial}{\partial \rho}\left(\rho \frac{\partial}{\partial \rho}\right)+\frac{1}{\alpha^{2} \rho^{2}} \frac{\partial^{2}}{\partial \theta^{2}}+\left(\frac{1-\alpha^{2}}{4 \alpha^{2}}\right) \frac{1}{\rho^{2}}-\left(\frac{1-\alpha}{\alpha}\right) \frac{\delta(\rho)}{\rho}\right] \psi=E \psi .
$$

On the other hand, the K-G theory yields

$$
-\frac{\hbar^{2}}{2 M}\left[\frac{1}{\rho} \frac{\partial}{\partial \rho}\left(\rho \frac{\partial}{\partial \rho}\right)+\frac{1}{\alpha^{2} \rho^{2}} \frac{\partial^{2}}{\partial \theta^{2}}-\left(\frac{1-\alpha}{\alpha}\right) \frac{\delta(\rho)}{\rho}\right] \psi=E \psi .
$$

Notice now that the contribution from an inverse squared distance interaction does not appear. This will lead to profound changes in the energy spectrum of particles on a cone.

Now, we decompose the Hilbert space $\mathcal{H}=L^{2}\left(\mathbb{R}^{2}\right.$ with respect to the angular momentum $\mathcal{H}=\mathcal{H}_{\rho} \otimes \mathcal{H}_{\theta}$, where $\mathcal{H}_{\rho}=L^{2}\left(\mathbb{R}^{+}, \rho d \rho\right)$ and $\mathcal{H}_{\theta}=L^{2}\left(\mathcal{S}^{1}, d \theta\right)$, with $\mathcal{S}^{1}$ denoting the unit sphere in $\mathbb{R}^{2}$. We have the fact that the $-\frac{\partial^{2}}{\partial \theta^{2}}$ is essentially self-adjoint in $L^{2}\left(S^{1}, d \theta\right)^{22,23}$. So, putting the wave function in the form

$$
\psi(\rho, \theta)=\Phi_{E}(\rho) e^{i l \theta},
$$

with $l \in \mathbb{Z}$, both Eqs. (14) and (15) can be written in a general form, that is,

$$
-\frac{\hbar^{2}}{2 M}\left[\frac{1}{\rho} \frac{\partial}{\partial \rho}\left(\rho \frac{\partial}{\partial \rho}\right)-\frac{\mu^{2}}{\rho^{2}}-\left(\frac{1-\alpha}{\alpha}\right) \frac{\delta(\rho)}{\rho}\right] \Phi_{E}=E \Phi_{E} .
$$


Note that in the Schrödinger theory it holds

$$
\mu^{2}=\frac{l^{2}}{\alpha^{2}}-\frac{\left(1-\alpha^{2}\right)}{4 \alpha^{2}}
$$

while

$$
\mu^{2}=\frac{l^{2}}{\alpha^{2}}
$$

in the $\mathrm{K}-\mathrm{G}$ theory. In the case of having a proficit angle, the relation (10) should be written as

$$
\alpha=\sinh \beta
$$

since in this case we must have $\alpha>1$. This is consistent with the fact that now the cone has a negative curvature, that is, the surface takes a saddle-like form. The mean curvature is now given by

$$
\mathrm{M}=\frac{\sqrt{\alpha^{2}-1}}{2 \alpha \rho}
$$

In this case, we have

$$
\mu^{2}=\frac{l^{2}}{\alpha^{2}}-\frac{\left(\alpha^{2}-1\right)}{4 \alpha^{2}} .
$$

In what follows, we replace the Gaussian curvature contribution to the potential by a shortranged potential supported inside a region with radius $a$, which is small as compared to the overall dimension of the system. This way, we have $U_{\text {short }}(\rho \geq a)=0$. The Schrödinger equation for the particle, in this case, is

$$
-\frac{\hbar^{2}}{2 M}\left[\frac{1}{\rho} \frac{\partial}{\partial \rho}\left(\rho \frac{\partial}{\partial \rho}\right)-\frac{\mu^{2}}{\rho^{2}}\right] \Phi_{E}+\frac{\hbar^{2}}{2 M} U_{\text {short }}(\rho) \Phi_{E}=E \Phi_{E},
$$

Here, $U_{\text {short }}(\rho)$ turns into the K of Eq. (11) in the limit $a \rightarrow 0$.

The Gaussian curvature is related to the topology of the cone, so is $U_{\text {short }}(\rho)$. Then, it can be modeled by boundary conditions 24 . Moreover, from the Gauss-Bonnet theorem, we have $^{25}$

$$
\iint_{s} \mathrm{~K} d A=\iint_{s} U_{\text {short }} d A=2 \pi(1-\alpha)
$$

This fact will be considered bellow. The problem (23) is replaced by

$$
-\frac{\hbar^{2}}{2 M}\left[\frac{1}{\rho} \frac{\partial}{\partial \rho}\left(\rho \frac{\partial}{\partial \rho}\right)-\frac{\mu^{2}}{\rho^{2}}\right] \Phi_{\eta, E}=E \Phi_{\eta, E}
$$

with $\Phi_{\eta, E}$ labeled by a parameter $\eta$ which is related to the behavior of the wave function in the limit $\rho \rightarrow a$. Next, we have to discover which boundary conditions are allowed to Eq. (17). This is performed via the the self-adjoint extension approach $\frac{23}{2} 26$. 
Before proceeding to find the solutions of Eq. (17), we will revise the bound states addressed in the Schrödinger theory. The details can be found in reference ${ }^{16}$.

\section{BOUND STATES - DA COSTA APPROACH}

We start by revising the bound states in the Schrödinger theory. For Eq. (17), two possible solutions rise up. The first one, corresponding to $\mu^{2} \leq 0$, is

$$
\Phi_{E}(\rho)=K_{i|\mu|}\left(\frac{\rho}{\hbar} \sqrt{-2 m E}\right)
$$

where $K_{\nu}(\rho)$ is the Bessel function of third kind, $|\mu|<1$, and the energies given by

$$
E=-\frac{2 \hbar^{2}}{M a^{2}} \exp \left[\frac{2}{\mu} \cot ^{-1}\left(\frac{1-\alpha}{\alpha \mu}-\frac{\mu}{2}\right)-2 \gamma_{e}\right]
$$

here $\gamma_{e}$ is the Euler-Mascheroni constant.

These results corresponds to $\alpha<1$. In this case, the mean curvature contributes attractively, while the Gaussian curvature yields a repulsive short-ranged potential. Eq. (18) implies that the only allowable value for the angular momentum is $l=0$, meaning that we have a single bound state. For $\alpha>1$, the relation (22) holds. The mean curvature contributes attractively again and the Gaussian curvature now leads to an attractive shortranged potential. We have a single bound state for $l=0$, as before. On the other hand, for $\mu^{2} \geq 0$, the wave solutions are

$$
\Phi_{E}(\rho)=K_{\mu}\left(\frac{\rho}{\hbar} \sqrt{-2 m E}\right)
$$

for $|\mu|<1$ and, the energies are given $b^{27}$

$$
E=-\frac{2 \hbar^{2}}{M a^{2}}\left[\frac{\Gamma(1+\mu)}{\Gamma(1-\mu)}\left(\frac{\mu^{2}+2\left(\frac{1-\alpha}{\alpha}\right)-2 \mu}{\mu^{2}+2\left(\frac{1-\alpha}{\alpha}\right)+2 \mu}\right)\right]^{1 / \mu}
$$

We have bound states for

$$
\begin{aligned}
& 1<\alpha \leq \sqrt{5} \text { at } l= \pm 1 \\
& \sqrt{17 / 5}<\alpha \leq \sqrt{17} \text { at } l= \pm 1 \text { and } \pm 2 \\
& \sqrt{37 / 5}<\alpha<\sqrt{37} \text { at } l= \pm 1, \pm 2, \text { and } \pm 3
\end{aligned}
$$

and so on.

In the next section, we solve the same problem in the K-G theory context, comparing the results with the ones discussed here. 


\section{BOUND STATES - SCHRÖDINGER EQUATION FROM THE K-G THEORY}

We now look for the bound states solving the Schrödinger equation which comes from the K-G theory. As discussed above, we can solve this problem using the self-adjoint extension approach. From Eq. (25), we have

$$
\mathcal{H} \Phi_{\eta, E}=\kappa^{2} \Phi_{\eta, E}
$$

where

$$
\mathcal{H}=-\frac{\hbar^{2}}{2 M}\left[\frac{1}{\rho} \frac{\partial}{\partial \rho}\left(\rho \frac{\partial}{\partial \rho}\right)-\frac{\mu^{2}}{\rho^{2}}\right] .
$$

Eq. (31) is the modified Bessel equation. In it, $\kappa^{2}=-2 M E / \hbar^{2}>0$, since we are looking for bound states.

Notice that, different from the Schrödinger theory, the inverse squared potential here is always attractive. This happens because, from Eq. (19), the only possible case is $\mu^{2} \geq 0$. For $\alpha<1$ (deficit angle), the Gaussian curvature is positive and the short ranged potential is repulsive, meaning that we have only scattering states. On the other hand, when $\alpha>1$ the Gaussian curvature is negative and such short ranged potential is negative, implying attractiveness. This is, therefore, the only case where bound states can exist.

This way, we can see the first difference between the two theories as follows. In the Schrödinger one, we can have bound states for either $\mu^{2}>0$ or $\mu^{2}<0$ (there is no possibility of having $\mu=0)$, regardless if we have a deficit $(\alpha<1)$ or a proficit $(\alpha>1)$ angle. For the $\mathrm{K}-\mathrm{G}$ theory the only possibility is for a proficit angle, since we have only the case $\mu^{2} \geq 0$. Note that this difference relies in the fact that the contribution from mean curvature is now absent in this theory.

We now proceed in order to find the bound states in the K-G theory. First of all, we must determine the full domain of $\mathcal{H}$ in $L^{2}\left(\mathcal{R}^{+}, \rho d \rho\right)$. In doing so, we have to find its deficient subspaces. This is done solving the eigenvalue equation

$$
\mathcal{H}^{\dagger} \Phi_{ \pm}= \pm i k_{0} \Phi_{ \pm}
$$

where $\mathcal{H}^{\dagger}=\mathcal{H}$. The general solution for this equation is given in terms of modified Bessel functions, denoted by $I_{\mu}$ and $K_{\mu}$. However, only $K_{\mu}$ is square integrable in all space. Then, 
we have as solutions of Eq. (33) the wave functions

$$
\Phi_{ \pm}(\rho)=\text { const. } K_{\mu}\left(\frac{\rho}{\hbar} \sqrt{\mp \varepsilon}\right)
$$

with $\mu^{2}<1$ and $\varepsilon=2 i M k_{0}$. The dimension of such deficient subspaces is $\left(n_{+}, n_{-}\right)=(1,1)$. Then, the wave functions $\Phi_{\eta, E}$ are written as

$$
\Phi_{\eta, E}(\rho)=\chi_{\mu}(\rho)+C\left[K_{\mu}\left(\frac{\rho}{\hbar} \sqrt{-\varepsilon}\right)+e^{i \eta} K_{\mu}\left(\frac{\rho}{\hbar} \sqrt{\varepsilon}\right)\right]
$$

where $\chi_{\mu}(\rho)$, with $\chi_{\mu}(a)=\dot{\chi}_{\mu}(a)=0$. The last term in Eq. (35) gives the correct behavior of the wave function when $\rho=a$. The parameters $\eta(\bmod 2 \pi)$ and $k_{0}$ represent the a priori choices of boundary conditions. The $U_{\text {short }}$ potential determines these parameters without ambiguity. This is done by finding a fitting formula for $\eta^{24}$ : we write $E=0$ or the static solution for the problem, that is

$$
-\frac{\hbar^{2}}{2 M}\left[\frac{1}{\rho} \frac{\partial}{\partial \rho}\left(\rho \frac{\partial}{\partial \rho}\right)-\frac{\mu^{2}}{\rho^{2}}\right] \Phi_{0}+\frac{\hbar^{2}}{2 M} U_{\text {short }}(\rho) \Phi_{0}=0 .
$$

Now, we require the continuity for the logarithmic derivative

$$
\left.\frac{\rho}{\Phi_{0}} \frac{d \Phi_{0}}{d \rho}\right|_{\rho=a}=\left.\frac{\rho}{\Phi_{\eta, 0}} \frac{d \Phi_{\eta, 0}}{d \rho}\right|_{\rho=a}
$$

where $\Phi_{\eta, 0}$ comes from Eq. (35) for $E=0$. The left-hand side of this equation can be achieved integrating (36) from 0 to $a$,

$$
-\int_{0}^{a} \frac{1}{\rho} \frac{d}{d \rho}\left(\rho \frac{d \Phi_{0}}{d \rho}\right) \rho d \rho+\int_{0}^{a} \Phi_{0} U_{\text {short }}(\rho) \rho d \rho+\mu^{2} \int_{0}^{a} \frac{\Phi_{0}}{\rho^{2}} \rho d \rho=0 .
$$

Now, considering that $\Phi_{0} / \rho^{2}$ does not change significantly in the range $[0, a]$, we have

$$
\int_{0}^{a} \frac{\Phi_{0}(\rho)}{r^{2}} \rho d \rho \approx \frac{\Phi_{0}(a)}{a^{2}} \int_{0}^{a} \rho d \rho=\frac{\Phi_{0}(a)}{2}
$$

From Eq. (24), we have

$$
\int_{0}^{a} \Phi_{0} U_{\text {short }}(\rho) \rho d \rho \approx \Phi_{0} \int_{0}^{a} U_{\text {short }}(\rho) \rho d \rho=\Phi_{0} \int_{0}^{a} \operatorname{K} \rho d \rho=\frac{(1-\alpha)}{\alpha} \Phi_{0}
$$

so that

$$
\left.\frac{a}{\Phi_{0}(a)} \frac{d \Phi_{0}(\rho)}{d r}\right|_{\rho=a}=\frac{(1-\alpha)}{\alpha}+\frac{\mu^{2}}{2} .
$$

Since $a \approx 0$, the right-hand side of Eq. (37) is calculated using the asymptotic representation for $K_{\mu}(x)$ in the limit $x \rightarrow 0$, given by

$$
K_{\nu}(x) \sim \frac{\pi}{2 \sin (\pi \nu)}\left[\frac{x^{-\nu}}{2^{-\nu} \Gamma(1-\nu)}-\frac{x^{\nu}}{2^{\nu} \Gamma(1+\nu)}\right] .
$$


Thus, taking into account (35), we arrive at

$$
\left.\frac{a}{\Phi_{\eta, 0}(a)} \frac{d \Phi_{\eta, 0}(\rho)}{d \rho}\right|_{\rho=a}=\left.\frac{1}{\Omega_{\eta}(a)} \frac{d \Omega_{\eta}(\rho)}{d \rho}\right|_{\rho=a}
$$

where

$$
\Omega_{\eta}(\rho)=\left[\frac{(\rho \sqrt{-\varepsilon})^{-\mu}}{2^{-\mu} \Gamma(1-\mu)}-\frac{(\rho \sqrt{-\varepsilon})^{\mu}}{2^{\mu} \Gamma(1+\mu)}\right]+e^{i \eta}\left[\frac{(\rho \sqrt{\varepsilon})^{-\mu}}{2^{-\mu} \Gamma(1-\mu)}-\frac{(\rho \sqrt{\varepsilon})^{\mu}}{2^{\mu} \Gamma(1+\mu)}\right]
$$

By inserting Eqs. (41) and (43) into Eq. (37), we find

$$
\left.\frac{1}{\Omega_{\eta}(a)} \frac{d \Omega_{\eta}(\rho)}{d \rho}\right|_{\rho=a}=\frac{(1-\alpha)}{\alpha}+\frac{\mu^{2}}{2},
$$

which gives us the parameter $\eta$ in terms of the physics of the problem, that is, the correct behavior of the wave functions when $\rho \rightarrow a$, or the coupling between the short-ranged potential $U_{\text {short }}(\rho)$ and the wave functions.

Next, we will find the bound states of the Hamiltonian $\mathcal{H}$ and using Eq. (45), the spectrum will be determined without any arbitrary parameter 28,29 . In doing so, we can go back to Eq. (25), whose general solution is

$$
\Phi_{\eta, E}(\rho)=K_{\mu}\left(\frac{\rho}{\hbar} \sqrt{-2 M E}\right)
$$

Since these solutions belong to the domain of $\mathcal{H}$, it has the form of Eq. (35), that is,

$$
\Phi_{\eta, E}(\rho)=\chi_{\mu}(\rho)+C\left[K_{\mu}\left(\frac{\rho}{\hbar} \sqrt{-\varepsilon}\right)+e^{i \eta} K_{\mu}\left(\frac{\rho}{\hbar} \sqrt{\varepsilon}\right)\right]
$$

for some $\eta$ selected from the physics of the problem. So, we substitute (46) in Eq. (35) and compute $a /\left.\Phi_{\eta, E}(a)\left(d \Phi_{\eta, E}(\rho) / d \rho\right)\right|_{\rho=a}$, using (42), achieving

$$
\left.\frac{a}{\Phi_{\eta, E}(a)} \frac{d \Phi_{\eta, E}(a)}{d \rho}\right|_{\rho=a}=\frac{\mu\left[a^{2 \mu} \Gamma(1-\mu)\left(-M E / \hbar^{2}\right)^{\mu}+2^{\mu} \Gamma(1+\mu)\right]}{a^{2 \mu} \Gamma(1-\mu)\left(-M E / \hbar^{2}\right)^{\mu}-2^{\mu} \Gamma(1+\mu)}=\frac{1}{\Omega_{\eta}(a)} \frac{d \Omega_{\eta}(a)}{d \rho} .
$$

By using Eq. (45) and solving the equation above for $E$, we find the energy spectrum

$$
E=-\frac{2 \hbar^{2}}{M a^{2}}\left[\frac{\Gamma(1+\mu)}{\Gamma(1-\mu)}\left(\frac{\mu^{2}+2\left(\frac{1-\alpha}{\alpha}\right)-2 \mu}{\mu^{2}+2\left(\frac{1-\alpha}{\alpha}\right)+2 \mu}\right)\right]^{1 / \mu}
$$

which is similar to that described in formula (29). The main difference is that now the effective angular momentum is just $\mu= \pm \frac{l}{\alpha}$ instead of the one given by Eqs. (18) and (22). As we noted, when $\alpha<1$, we have $U_{\text {short }}>0$, that is, there is no bound states in this case. On the other hand, if $\alpha>1, U_{\text {short }}<0$ and bound states can show up. Because of 
the condition $|\mu|<1$, the value of the proficit angle will determine which values of angular momentum are allowed. For example, if $\alpha=1.5$, then we must have $l=-1,0,1$. If $\alpha=2.1$, then $l$ admits the values $-2,-1,0,1,2$, and so on. Now, we summarize the results of this section, that is,

$$
\begin{aligned}
& 1<\alpha \leq 2 \text { at } l=0, \pm 1 \\
& 2<\alpha \leq 3 \text { at } l=0, \pm 1 \text { and } \pm 2 \\
& 3<\alpha \leq 4 \text { at } l=0, \pm 1, \pm 2, \text { and } \pm 3
\end{aligned}
$$

and so on.

Notice that the dependence of the bound states on the angular momentum $l$ changes significantly when compared to the dependence observed in the Schrödinger theory. For example, the null angular momentum, $l=0$, now can exist.

Concluding this section, when $\mu^{2}>0$ and $\alpha>1$, the mean curvature potential is repulsive and the short-ranged potential, due to the Gaussian curvature, is attractive in the Schrödinger theory. On the other hand, in the K-G theory, the absence of the mean curvature potential affects drastically the bound states of a quantum particle on a cone. In both cases, the Gaussian curvature is the sole responsible for the bound states.

\section{CONCLUDING REMARKS}

In a previous work ${ }^{16}$, the quantum dynamics of a particle on a cone was addressed in the context of the Schrödinger theory considering the da Costa's approach. In this work we investigate the bound states of a quantum particle on the same surface within the K-G theory framework. In both theories, the conical geometry provides a $\delta$-function interaction which can be either attractive or repulsive, depending on the cone parameter $\alpha$. However, in the first theory, the conical geometry introduces an inverse squared distance potential due to the mean curvature which leads to an effective potential that can be either attractive or repulsive, depending on $\alpha$. This mean curvature term does not show up when we study such system using the K-G theory. We saw in the previous sections that this difference implies profound changes for the dependence of the bound states on the angular momentum l. These results are summarized in Tables \and [I.

From Table I, we see that, for $\alpha>1$, the attractive short-ranged potential (Gaussian curvature) guarantees the existence of bound states. For $\alpha<1$ (repulsive short-ranged 


\begin{tabular}{lcc}
\hline \hline & $\alpha>1$ & $\alpha<1$ \\
\hline$\mu^{2}<0$ & 1 bound state for $l=0$ & 1 bound state for $l=0$ \\
$\mu^{2}>0$ & bound states & scattering states for $l \neq 0$ \\
\hline \hline
\end{tabular}

TABLE I. Summary of the results - Schrödinger Theory

\begin{tabular}{lcc}
\hline \hline & $\alpha>1$ & $\alpha<1$ \\
\hline$\mu^{2} \geq 0$ & bound states & scattering states \\
\hline \hline
\end{tabular}

TABLE II. Summary of the results - K-G Theory

potential), an attractive effective potential yields a bound state for $l=0$. However, for $l \neq 0$, we have only repulsive potentials and no bound states appear.

In Table II, the absence of the mean curvature makes the inverse square potential always repulsive, so the Gaussian curvature is the unique responsible for the existence of bound states. They exist only when we have a proficit angle $\alpha$, that is, $\alpha>1$. No bound states appear in the actual cone $(\alpha<1)$. This is in great contrast with the results which comes from the Schrödinger theory.

In order to investigate the behavior of fermions in two dimensions, it is important to explore phenomena like the quantum Hall effect, magnetization and persistent currents in two dimensional semiconductors. For a cone, these two last physical quantities were investigated in reference ${ }^{30}$. The authors did not take into account either $V_{\text {daCosta }}$ or $V_{\text {geo }}$. But their results are correct within the K-G theory, since no potential containing the mean curvature of a cone appears and the electrons are localized on a ring far from the cone apex. However, if we consider the Schrödinger theory with the da Costa's approach, the effective angular momentum now depends on an extra piece which comes from $V_{\text {daCosta }}$, as we saw above. So, measurements on the persistent current on conical surfaces can help to decide which theory is suitable to describe fermions in two dimensions. Perhaps, both theories can be used depending on which material we have in hands. We must mention do not not apply to a monolayer graphene, where fermions are in fact described by a massless Dirac theory. Although, they could be important for quantum systems in two dimensional surfaces consisting of more than one layer, as a bilayer graphene sheet. 
As a final word, it is clear that both theories provide, theoretically, contradictory physical results. We have discussed this difference here, for quantum particles on a conical surface. The K-G theory can also change the scenario when we consider possible applications to a bilayer graphene.

\section{ACKNOWLEDGMENTS}

The authors would like to thank M. M. Ferreira Jr. for critical reading the manuscript. E. O. Silva acknowledges research grants by CNPq-(Universal/2011) and C. Filgueiras by CAPES (Nanobiotec)/CNPq(Universal/2011).

\section{REFERENCES}

${ }^{1}$ L. I. Magarill, A. V. Chaplik, and M. V. Entin, Phys. Usp. 48, 953 (2005).

${ }^{2}$ C. L. Foden, M. L. Leadbeater, and M. Pepper, Phys. Rev. B 52, R8646 (1995).

${ }^{3}$ A. Chaplik, L. Magarill, and D. Romanov, Physica B 249251, 377 (1998).

${ }^{4}$ A. B. Vorob'ev, K.-J. Friedland, H. Kostial, R. Hey, U. Jahn, E. Wiebicke, J. S. Yukecheva, and V. Y. Prinz, Phys. Rev. B 75, 205309 (2007).

${ }^{5}$ G. Ferrari, A. Bertoni, G. Goldoni, and E. Molinari, Phys. Rev. B 78, 115326 (2008).

${ }^{6}$ D. Bulaev and V. Margulis, Eur. Phys. J. B 36, 183 (2003).

${ }^{7}$ M. V. Entin and L. I. Magarill, Phys. Rev. B 66, 205308 (2002).

${ }^{8}$ M. Encinosa, Phys. Rev. A 73, 012102 (2006).

${ }^{9}$ C. Filgueiras and B. de Oliveira, Ann. Phys. (Berlin) 523, 898 (2011).

${ }^{10}$ R. C. T. da Costa, Phys. Rev. A 23, 1982 (1981).

${ }^{11}$ G. Ferrari and G. Cuoghi, Phys. Rev. Lett. 100, 230403 (2008).

${ }^{12}$ Y. N. Joglekar and A. Saxena, Phys. Rev. B 80, 153405 (2009).

${ }^{13}$ V. Atanasov, R. Dandoloff, and A. Saxena, Phys. Rev. B 79, 033404 (2009).

${ }^{14}$ R. Dandoloff, A. Saxena, and B. Jensen, Phys. Rev. A 81, 014102 (2010).

${ }^{15}$ B. Jensen and R. Dandoloff, Phys. Lett. A 375, 448 (2011).

${ }^{16}$ C. Filgueiras and F. Moraes, Ann. Phys. (N.Y.) 323, 3150 (2008).

${ }^{17}$ M. Katanaev and I. Volovich, Ann. Phys. (N.Y.) 216, 1 (1992).

${ }^{18}$ S. Azevedo and F. Moraes, J. Phys.: Condens. Matter 12, 7421 (2000). 
${ }^{19}$ J. A. Miranda and F. Moraes, J. Phys. A 36, 863 (2003).

${ }^{20} \mathrm{~V}$. Osipov and E. Kochetov, JETP Lett. 73, 562 (2001), 10.1134/1.1387528.

${ }^{21}$ A. M. de M. Carvalho, C. Sátiro, and F. Moraes, Europhys. Lett. 80, 46002 (2007).

${ }^{22}$ K. Kowalski, K. Podlaski, and J. Rembieliński, Phys. Rev. A 66, 032118 (2002).

${ }^{23}$ M. Reed and B. Simon, Methods of Modern Mathematical Physics. II. Fourier Analysis, Self-Adjointness. (Academic Press, New York - London, 1975).

${ }^{24}$ B. S. Kay and U. M. Studer, Commun. Math. Phys. 139, 103 (1991).

${ }^{25}$ M. Hayashi, Phys. Lett. A 342, 237 (2005).

${ }^{26}$ G. Bonneau, J. Faraut, and G. Valent, Am. J. Phys. 69, 322 (2001).

${ }^{27}$ These results are different from those in reference ${ }^{16}$ since in equation (37) in this reference has a sign mistake.

${ }^{28}$ F. M. Andrade, E. O. Silva, and M. Pereira, Phys. Rev. D 85, 041701(R) (2012).

${ }^{29}$ C. Filgueiras, E. O. Silva, W. Oliveira, and F. Moraes, Ann. Phys. (N.Y.) 325, 2529 (2010).

${ }^{30}$ C. Furtado, A. Rosas, and S. Azevedo, Europhys. Lett. 79, 57001 (2007). 